\title{
O método BiblioGrad para avaliação de acervos de livros de graduação: instrumento para gestão de recursos para aquisição em um sistema de bibliotecas universitárias
}

\author{
Letícia Strehl \\ Bibliotecária da Universidade Federal do Rio Grande do Sul, \\ Biblioteca Central - Porto Alegre, RS - Brasil \\ E-mail: leticia.strehl@ufrgs.br
}

\section{June Magda Rosa Scharnberg}

Bibliotecária-chefe da Escola de Engenharia. Universidade Federal do Rio Grande do Sul - Porto Alegre, RS - Brasil E-mail: june@ufrgs.br

\section{Zaida Horowitz}

Analista de sistemas do Centro de Processamento de Dados. Universidade Federal do Rio Grande do Sul - Porto Alegre, RS - Brasil

E-mail: zaida@cpd.ufrgs.br

\section{Viviane Carrion Castanho}

Diretora da Biblioteca Central da Universidade Federal do Rio Grande do Sul - Porto Alegre, RS - Brasil

E-mail: vivicc@ufrgs.br

\section{Resumo}

O artigo apresenta um método criado no âmbito da Biblioteca Central da Universidade Federal do Rio Grande do Sul para a avaliação de acervos de livros que subsidiam as atividades de ensino de graduação. Descreve a fase de montagem do sistema de informações necessário para a elaboração do relatório de avaliação dos acervos. E, por fim, apresenta como os dados do relatório são congregados numa fórmula proposta com a finalidade de distribuir recursos para compra de livros de graduação entre as unidades do sistema de bibliotecas.

\section{Palavras-chave}

Avaliação de coleções de livros de graduação. Fórmulas de alocação de recursos.

Ci. Inf., Brasília, DF, v. 39 n. 3, p.105-115, set./dez., 2010

\begin{abstract}
The BiblioGrad method for evaluating the collections of undergraduate books: instrument for managing the acquisition resources in university library systems
\end{abstract}

\begin{abstract}
This paper presents a method elaborated in the context of the Central Library of the Universidade Federal do Rio Grande do Sul for the evaluation of the book collections which give support to the undergraduate teaching activities. It describes the structuring phase of the information system for the elaboration of the collection evaluation report. And lastly, it shows how the report data are joined in a formula for allocating funds for the purchase of undergraduate books among the library system units.
\end{abstract}

Keywords

Evaluation of undergraduate book collections. Fund allocation formulas.

\section{INTRODUÇÃO}

O gerenciamento dos processos que envolvem o desenvolvimento de coleções é complexo por natureza. Quando analisado e operacionalizado no âmbito de um sistema de bibliotecas, as dificuldades inerentes passam a ser potencializadas pelo número de unidades envolvidas e pela diversificação das características dos usuários do sistema.

Podemos destacar que o desenvolvimento de coleções em bibliotecas de grandes universidades enfrenta uma parte significativa dos maiores desafios do processo, considerando o tamanho dos acervos, a heterogeneidade da comunidade atendida e o dinamismo do campo científico. Diante disso, observamos que, no âmbito da Biblioteca Central (BC) da Universidade Federal do Rio Grande do Sul, 
precisávamos dispor de novos instrumentos para auxiliar na tarefa de administração dos recursos para investimento nos acervos do Sistema de Bibliotecas da Universidade (SBU).

Nos últimos anos, com o expressivo aumento dos recursos destinados à atualização de acervo, a direção da $\mathrm{BC}$ passou a investir maciçamente em livros que subsidiam as atividades de ensino de graduação. Entretanto, a tarefa de determinação da demanda por livros didáticos para distribuição dos recursos entre as bibliotecas do SBU de forma proporcional à demanda a ser atendida tem se tornado ainda mais complexa, principalmente em função da ampliação do número de vagas em cursos de graduação, da diversificação das áreas de formação, das constantes mudanças curriculares e da diminuição relativa dos recursos disponíveis para o financiamento do sistema público de ensino.

Frequentemente, a direção da BC utilizava critérios quantitativos para distribuição dos recursos entre as 33 diferentes bibliotecas do SBU. Neste enfoque, as informações como quantidade de usuários, volume de circulação de materiais, tamanho do acervo e número de cursos de graduação oferecidos pela(s) unidade(s) de ensino a que a biblioteca se subordina poderiam ser parâmetros determinantes para definição dos recursos disponibilizados.

Entretanto, algumas destas variáveis apresentavam uma capacidade de percepção limitada para medir a demanda a ser atendida pelas bibliotecas, principalmente quando:

a) os recursos eram destinados para uma biblioteca de acordo com o número de alunos de graduação atendidos nos curso de sua unidade, desconsiderando a demanda gerada por estes alunos que era atendida em bibliotecas de outras unidades ${ }^{1}$;

\footnotetext{
${ }^{1}$ O Instituto de Matemática da UFRGS, por exemplo, possui 663 alunos nos cursos por ele oferecidos. Todavia, apenas na disciplina de "Cálculo e geometria analítica I - A" ministrada pelo instituto a vários cursos de outras unidades de ensino são atendidos 1.269 alunos. Dada a organização das turmas desta disciplina na UFRGS, todos estes alunos devem ser atendidos pela Biblioteca do Instituto de Matemática.
}

b) a demanda era estimada pelo volume de circulação, valorizando o uso real em detrimento do potencial. Adotado este critério, bibliotecas com acervos mais deficientes tinham sua demanda subestimada pelas necessidades que não conseguiam suprir e não pelo atendimento que deveriam prestar;

c) a carência dos acervos era deduzida das informações de uso e de reservas feitas aos itens bibliográficos, desconsiderando a demanda pelos materiais que não constituem os acervos.

Nestas circunstâncias, verificamos que o dimensionamento dos recursos necessários para o atendimento das demandas das comunidades das diversas bibliotecas deveria ser feito com base em dados mais específicos de acervo e demanda potencial, envolvendo critérios qualitativos e quantitativos de análise.

Tendo em vista este propósito, foi idealizado um método de avaliação de acervos de livros didáticos denominado BiblioGrad. O BiblioGrad parte das informações constantes na bibliografia dos planos de ensino das disciplinas de graduação e estrutura um sistema de informações para:

a) verificar a disponibilidade de cada um dos títulos nos acervos;

b) identificar a demanda potencial por títulos específicos a ser atendida pelas diversas bibliotecas;

c) diagnosticar a situação dos acervos de livros para graduação do SBU;

d) subsidiar a tomada de decisões sobre a distribuição dos recursos entre as bibliotecas de forma proporcional às carências de acervo identificadas;

e) oferecer às bibliotecas do SBU um instrumento para seleção dos livros a serem adquiridos, visando ao acervo de graduação.

Nas seções a seguir são descritos os elementos que constituíram a formulação e a aplicação do BiblioGrad no SBU, iniciando pela fase de

Ci. Inf., Brasília, DF, v. 39 n. 3, p.105-115, set./dez., 2010 
O método BiblioGrad para avaliação de acervos de livros de graduação: instrumento para gestão de recursos para ...

montagem do sistema de informações, passando pela elaboração do relatório de avaliação dos acervos, e finalizando com a exposição dos usos atribuídos ao instrumento para a gestão dos recursos pela BC. Nas considerações finais, mostramos as perspectivas de desenvolvimento futuro do BiblioGrad, principalmente no que diz respeito à criação de instrumentos para auxiliar na seleção dos livros a adquirir pelas bibliotecas setoriais.

Pretendemos com este texto contribuir para a discussão sobre a gestão de acervos em bibliotecas universitárias brasileiras, a exemplo de outros relatos (LUBISCO, 2008; VICENTINI, 2006; USP, 1998; DIAS; PIRES, 2003; GONZAGA FILHA, 2006), bem como oferecer os subsídios necessários para o uso do método em outros contextos aos quais possa interessar.

\section{ESTRUTURAÇÃO DE SISTEMA PARA A COLETA DAS INFORMAÇÕES: CONGREGANDO BANCOS DE DADOS COM O USO DO MARC}

Os planos de ensino das disciplinas são um importante instrumento para a implementação dos projetos pedagógicos dos cursos de graduação (BRASIL, 2008). Para os bibliotecários de bibliotecas universitárias, as bibliografias citadas nos planos constituem uma ferramenta para seleção de documentos para aquisição, funcionando, por definição, como bibliografias padrão e listas de livros recomendados específicos (ad hoc list) para o desenvolvimento das atividades de ensino de graduação (FIGUEIREDO, 1998).

Tendo à disposição este relevante recurso, o BiblioGrad foi criado para verificar, em consonância com avaliações institucionais promovidas pelo MEC (BRASIL, 2008), em que medida o SBU possui materiais adequados, em quantidade, pertinência acadêmico-científica e atualização, para implementação do projeto pedagógico dos cursos a que as bibliotecas oferecem suporte.

Para tanto, verificamos que o BiblioGrad deveria constituir um sistema capaz de congregar as já conhecidas informações sobre os acervos das bibliotecas com os dados específicos das atividades acadêmicas de graduação.

Todavia, na UFRGS, a manipulação destas informações é ainda dificultada pela inexistência de um banco de dados que sistematize os conteúdos dos planos de ensino. Assim, a coleta dos planos passou a ser feita junto aos departamentos, individualmente, ficando a organização das informações condicionadas à sistemática de cada um dos setores. Existem bibliotecas que precisam trabalhar com até oito (8) departamentos, cada qual com sua forma de organização. Após a obtenção dos planos de ensino pelas bibliotecas, a associação dos dados acadêmicos com os de acervo ficou dependente de uma solução a ser encontrada. Para tanto, trabalhamos com o Centro de Processamento de Dados da UFRGS (CPD) no sentido de criar um elo entre os dois bancos de dados da Universidade que contêm as informações necessárias: o Sistema de Graduação e o Sistema de Automação de Bibliotecas (SABi).

Como o SABi utiliza o formato MARC (MAchineReadable Cataloging) para o registro bibliográfico e de autoridades, e a estrutura deste formato permite a criação de campos próprios para satisfação de necessidades institucionais (bloco 9XX), criamos um campo para ser preenchido no registro bibliográfico dos livros citados nos planos de ensino, de modo a identificar as disciplinas de graduação em que eles são adotados.

Os campos criados foram o 902, no formato de registro bibliográfico, e o 192, no formato de registro de autoridades. Os campos contêm o nome das disciplinas cujos planos citam as obras catalogadas para estabelecer o vínculo necessário entre os dados do SABi e do Sistema de Graduação.

Basicamente, o campo 192 do formato de registro de autoridades foi criado para facilitar o preenchimento do campo 902 em registros bibliográficos e para diminuir a ocorrência de inconsistências na entrada dos dados de disciplinas. 
Os registros de autoridades contendo as informações necessárias das disciplinas são preenchidos automaticamente, via sistema, pela gerência do SABi a partir dos dados registrados no Sistema de Graduação. Estes registros de autoridades não podem ser criados por bibliotecários do SBU e são alterados pela gerência do SABi à medida que os dados de disciplinas sofrem alterações, dispensando rotinas manuais de correção.

O campo 192 contém o nome, a sigla e o código de disciplina de cursos de graduação da UFRGS e contempla exatamente os dados que individualizam uma dada disciplina no Sistema de Graduação (figura 1).

O campo 902 a ser preenchido nos registros bibliográficos de livros utilizados em atividades de ensino de graduação contempla, além das informações contidas nos três subcampos do 192, os dados relacionados especificamente à forma de citação da obra no plano de ensino: status da bibliografia (básica ou complementar), parte e volume citados. A figura 2 mostra um registro bibliográfico do SABi com o campo 902 preenchido, indicando, por exemplo, que o livro Introduction to quantum mechanics, escrito por Griffiths, foi citado no plano de ensino da disciplina "Tópicos avançados em física II".

Implementada a estrutura para coleta dos dados sobre os livros utilizados em cursos de graduação, as bibliotecas do SBU reúnem os planos de ensino das disciplinas oferecidas dos departamentos de sua(s) unidade(s) correspondente(s) e identificam

\section{FIGURA 1}

Exemplo de registro de autoridade de uma disciplina de graduação

\begin{tabular}{|c|c|}
\hline Lider & DR $\quad \mid \cdots n z \cdots \cdots \cdots \cdot \cdots \cdots$ \\
\hline Campo controle & - 051020 nn-acnnnaaan-.........-un-ana-...-.d \\
\hline sciplina Grad & 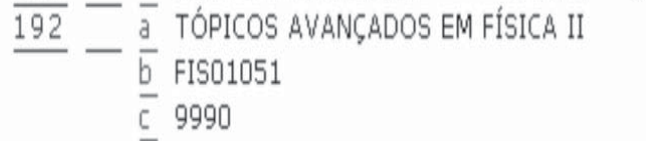 \\
\hline
\end{tabular}

\section{FIGURA 2}

Registro bibliográfico de livro citado em plano de ensino de disciplina de curso de graduação

\begin{tabular}{|c|c|c|c|c|}
\hline Lider & EDR & . & - & 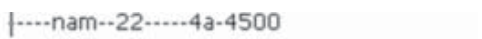 \\
\hline Campo controle & 008 & - & - & $00101052005 \cdots x x$..............001-0-eng-d \\
\hline ISBN & $\overline{020}$ & & $\bar{a}$ & 0131118927 \\
\hline Fonte catalog. & $\overline{040}$ & - & $\bar{a}$ & $\begin{array}{l}\text { BIPaURS } \\
\text { por }\end{array}$ \\
\hline Autor pessoal & 100 & 1 & $\frac{\bar{a}}{4}$ & $\begin{array}{l}\text { Griffiths, David Jeffrey } \\
\text { aut }\end{array}$ \\
\hline Titulo & 245 & 10 & & $\begin{array}{l}\text { Introduction to quantum mechanics } \\
\text { David J. Griffiths }\end{array}$ \\
\hline Área public. & 260 & & $\frac{\bar{a}}{\bar{b}}$ & $\begin{array}{l}\text { Upper Saddle River, N.J. } \\
\text { Prentice Hall } \\
\text { c2005. }\end{array}$ \\
\hline Desc. física & 300 & 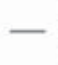 & $\overline{\mathrm{a}}$ & $\begin{array}{l}\text { ix, } 468 \mathrm{p} \text {. } \\
\text { ii. }\end{array}$ \\
\hline Assunto & 650 & 04 & $\bar{a}$ & Equacoes de ondas \\
\hline Assunto & $\overline{650}$ & $\overline{04}$ & $\bar{a}$ & Equacao de schrodinger \\
\hline Assunto & $\overline{650}$ & $\overline{04}$ & $\bar{a}$ & Teoria quantica \\
\hline Assunto & $\overline{650}$ & $\overline{04}$ & $\bar{a}$ & Teoria da perturbacao \\
\hline Assunto & $\overline{650}$ & $\overline{04}$ & $\bar{a}$ & Espalhamento \\
\hline Macrodescritor & $\overline{690}$ & & $\bar{a}$ & Fisica \\
\hline Disciplina grad & 902 & - & $\frac{\frac{a}{b}}{\frac{c}{d}}$ & $\begin{array}{l}\text { TÓPICOS AVANCADOS EM FÍSICA II } \\
\text { FISO1051 } \\
9990 \\
\text { Bibliografia básica }\end{array}$ \\
\hline
\end{tabular}

no SABi os registros bibliográficos das obras citadas $^{2}$.

Para facilitar a consulta aos dados de disciplinas informados nos registros bibliográficos dos livros, foram criados no SABi índices de busca específicos para este fim e um relatório (figura 3) que possibilita às bibliotecas controlar todos os livros identificados como bibliografia de graduação, por curso ou departamento.

Atualmente, existem no SABi 5.864 registros de autoridades para as disciplinas de graduação e 12.902 registros bibliográficos definidos como livros de graduação.

Findo o processo de identificação executado pelas bibliotecas, a BC passou a dispor dos dados necessários para realização da avaliação dos acervos de livros de graduação do SBU. A seção a seguir descreve os critérios estabelecidos como parâmetros de avaliação do acervo e o relatório que constitui o principal produto do BiblioGrad.

\footnotetext{
2 As instruções com os procedimentos operacionais a serem adotados pelos bibliotecários para a aplicação do BiblioGrad são abordadas em cursos específicos para este fim. O material utilizado está disponível em: http://www.biblioteca.ufrgs.br/modulo8-3.ppt (STREHL; CASTANHO, 2007).
}

Ci. Inf., Brasília, DF, v. 39 n. 3, p.105-115, set./dez., 2010 
O método BiblioGrad para avaliação de acervos de livros de graduação: instrumento para gestão de recursos para ...

FIGURA 3

Telas que compõem a interface do sistema de geração do relatório que identifica os livros utilizados nas disciplinas de graduação

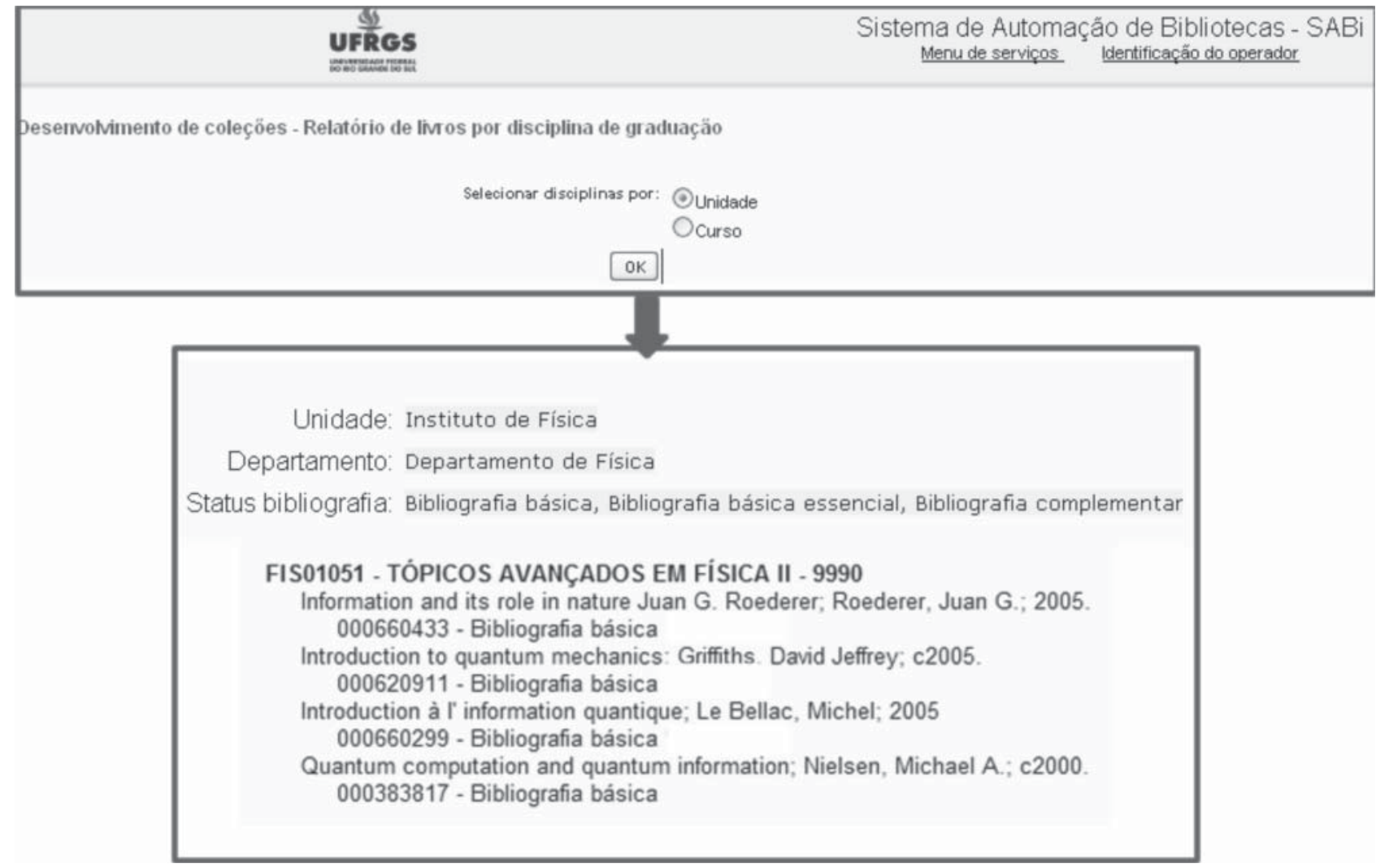

DEFINIÇÃO DOS PARÂMETROS DE AVALIAÇÃO E ELABORAÇÃO DO RELATÓRIO

A literatura de desenvolvimento de coleções sobre critérios de avaliação de acervos e fórmulas para alocação de recursos em bibliotecas é vasta e, entre outras funções, contribui para a realização da tarefa de distribuição de orçamentos mais limitados do que as demandas das comunidades usuárias.

Excelentes artigos de revisão foram publicados sobre o tema, sintetizando as principais questões envolvidas no processo; dentre eles, destacamos os trabalhos escritos por Budd (1991), Walters (2007) e Canepi (2007). Estes textos apresentam as inúmeras variáveis contempladas em métodos utilizados em grandes bibliotecas, oferecendo um panorama geral dos aparatos disponíveis para gestão de coleções.
Entretanto, mesmo com o avanço da área em nível internacional, observamos grande dificuldade de aplicação das técnicas apresentadas ao contexto brasileiro. Dentre os problemas enfrentados, além das flagrantes diferenças de capacidade de investimento das instituições oriundas dos países desenvolvidos, verificamos a falta de estatísticas globais que possam subsidiar o estabelecimento de parâmetros de análise aplicáveis às unidades e sistemas de informação nacionais, mesmo no que tange às bibliotecas universitárias, tradicionalmente mais integradas do que os outros tipos de bibliotecas no Brasil.

A quantidade de aspectos passíveis de compor um modelo para a tomada de decisões depende dos subsídios de que dispomos para avaliar os elementos eleitos como critérios. Portanto, o sentido das fórmulas tratadas na literatura internacional está vinculado aos padrões de desempenho dos produtos e serviços prestados pelas instituições envolvidas 
e desconhecemos em que nível eles se aplicam fora desse contexto, em conjunturas econômicas e culturais diversas.

Iniciativas como a do Library Statistics Program ${ }^{3}$ (do National Center for Education Statistics), que congregam dados de diversos sistemas e oferecem uma gama de parâmetros para realização de comparações, são indispensáveis para que possamos delinear modelos de distribuição de recursos pautados em um complexo quadro de referência. Dizemos complexo, uma vez que as variáveis específicas vistas de modo isolado apresentam uma capacidade limitada de representação da realidade e que a composição de um modelo multivariado depende das informações disponíveis sobre os vários aspectos.

Mesmo com todas as dificuldades advindas da falta de dados, um elemento indispensável a que não conseguimos nos furtar de trabalhar foi a demanda potencial estimada. Na tentativa de buscar um parâmetro que identificasse esse aspecto, trabalhamos com os padrões indicados no instrumento de avaliação dos cursos de graduação do MEC (BRASIL, 2008), que estabelece que uma biblioteca em condições ótimas disponibiliza em seu acervo a seguinte quantidade de exemplares:

a) bibliografia básica: um (1) exemplar para oito (8) alunos;

b) bibliografia complementar: um (1) exemplar por disciplina.

Estabelecidos os critérios de estimativa da demanda potencial, que, pela inexistência de estudos, não sabemos se são os ideais, mas que são os oficiais, foi desenvolvido um sistema de informações para agregar os dados necessários e fornecer um relatório de avaliação. Desse modo, para a análise das condições dos acervos do SBU, o instrumento parte da informação sobre cada obra/volume "j" citado no plano de ensino da(s) disciplina(s) ministrada(s) pelo(s) departamento(s) $\mathrm{da}(\mathrm{s})$ unidade(s) atendidas pela biblioteca "i", como exemplifica a tabela 1 .

De acordo com esses dados, as bibliotecas de engenharia e de administração precisam do livro Fundamentos da administração da produção, escrito por Davis. A administração, mesmo tendo em

\section{TABELA 1}

Estrutura do relatório de avaliação dos acervos de livros didáticos de graduação do SBU

\begin{tabular}{|c|c|c|c|c|c|c|c|c|c|c|c|c|}
\hline Item & Referência & Vol. & Bib. & $\begin{array}{c}\text { Disciplinas } \\
\text { BB }\end{array}$ & $\begin{array}{c}\text { N. Alunos } \\
\text { BB }\end{array}$ & $\begin{array}{l}\text { N. exe. } \\
\text { ideal BB }\end{array}$ & $\begin{array}{l}\text { Disciplinas } \\
\text { BC }\end{array}$ & $\begin{array}{c}\text { N. Disc. } \\
\text { BC }\end{array}$ & $\begin{array}{l}\text { N. exe. } \\
\text { ideal BC }\end{array}$ & $\begin{array}{l}\text { N. exe. } \\
\text { ideal total }\end{array}$ & $\begin{array}{l}\text { N. exe. } \\
\text { Bib. }\end{array}$ & $\begin{array}{c}\text { Carência } \\
\text { biblioteca }\end{array}$ \\
\hline & $\mathbf{j}$ & & i & & f & h & & $\mathrm{g}$ & d & $\mathrm{t}$ & e & $\mathrm{p}$ \\
\hline 1 & $\begin{array}{l}\text { Davis, Mark M. } \\
\text { Fundamentos da } \\
\text { administração da } \\
\text { produção. } 3 \text {. ed. Porto }\end{array}$ & & Administração & 8406 & 219 & 27 & & 0 & 0 & 27 & 12 & 15 \\
\hline 2 & $\begin{array}{l}\text { Davis, Mark M. } \\
\text { Fundamentos da } \\
\text { administração da } \\
\text { produção. 3. ed. Porto }\end{array}$ & & Engenharia & 9163,9167 & 61 & 8 & & 0 & 0 & 8 & 2 & 6 \\
\hline 3 & $\begin{array}{l}\text { A voz do especialista } \\
\text { Rio de Janciro: Revinter. }\end{array}$ & & Medicina & & 0 & 0 & 16998 & 1 & 1 & 1 & 0 & 1 \\
\hline 4 & $\begin{array}{l}\text { A voz do especialista } \\
\text { Rio de Janciro: Revinter. }\end{array}$ & 1 & Medicina & & 0 & 0 & 16998 & 1 & 1 & 1 & 0 & 1 \\
\hline 5 & $\begin{array}{l}\text { Guirado, M.C. } \\
\text { Reportagem: a arte da } \\
\text { investigação. São Paulo: }\end{array}$ & 2 & Comunicação & 2088,2100 & 48 & 6 & 2099 & 1 & 1 & 7 & 2 & 5 \\
\hline
\end{tabular}

${ }^{3}$ http://nces.ed.gov/surveys/libraries/index.asp 
O método BiblioGrad para avaliação de acervos de livros de graduação: instrumento para gestão de recursos para ...

sua coleção mais exemplares do título do que a engenharia, precisa adquirir um número maior de itens para suprir suas carências porque a demanda potencial estimada para esta obra nesta biblioteca é muito maior, 219 em comparação a 61 alunos.

Além da identificação de deficiências quantitativas de exemplares de determinado título em uma dada biblioteca, o instrumento procura evidenciar as lacunas qualitativas da coleção. É o caso dos volumes 1 e 2 de $A$ voz do especialista, na biblioteca de medicina. Para estes volumes, a aquisição de apenas um exemplar seria suficiente para o atendimento da demanda potencial estimada, o que quantitativamente não os torna uma prioridade. Todavia, a indisponibilidade completa da informação no acervo poderá inverter a ordem dos critérios de seleção e transformá-la em uma aquisição urgente.

Em síntese, o que pretendemos com o relatório é mapear a situação do acervo de cada una das unidades, biblioteca "i", para atendimento da demanda potencial a um título, obra “j”, partindo do número ideal de exemplares a constituir os vários acervos e os materiais efetivamente disponíveis. Estes parâmetros foram medidos da seguinte forma:

Fórmula para identificação do número ideal de exemplares (1)

$$
t_{j i}=\frac{f_{j i}}{8}+d_{j i}
$$

Onde:

$t_{j i}$ é o número ideal de exemplares da obra “j”" para atendimento da demanda potencial pela biblioteca " $i$ ";

$f_{j i}$ é o número de alunos matriculados na(s) disciplina(s) que utilizam a obra “j”" como bibliografia básica;

$d_{j i}$ é o número de disciplinas que utilizam a obra "j”" como bibliografia complementar
Fórmula para identificação de carências de acervo (2)

$p_{j i}=t_{j i}-e_{j i}$

Onde:

$p_{j i}$ é o número de exemplares da obra “j”" carentes no acervo da biblioteca " $i$ " para o atendimento da demanda potencial;

$e_{j i}$ é o número de exemplares da obra “j”” existentes no acervo da biblioteca " $i$ ".

Trabalhamos na próxima seção as aplicações deste relatório nas atividades de desenvolvimento de coleções do SBU.

\section{USO DOS RESULTADOS DA AVALIAÇÃO PARA A DISTRIBUIÇÃO DE RECURSOS ENTRE AS BIBLIOTECAS}

Com o BiblioGrad, a BC passou a dispor de informações sobre a situação dos acervos do SBU, partindo de uma previsão de demanda potencial a ser atendida nas diferentes bibliotecas. Um dos principais usos do método pela $\mathrm{BC}$ é como instrumento de gestão dos recursos para a aquisição de livros. Na prática, tendo em vista a necessidade de distribuição de um orçamento específico, os dados sobre as carências de exemplares de obras específicas (tabela 1) são consolidados por biblioteca, traçando um esquema geral da situação das coleções.

Estas informações formam um segundo relatório que congrega os parâmetros para a realização de uma distribuição de recursos na qual cada biblioteca receberá um montante diretamente proporcional ao preço médio dos livros de sua área e às carências existentes em seu acervo.

Todos estes aspectos congregados levaram à elaboração de uma fórmula para distribuição de recursos para aquisição de livros didáticos de graduação, como se descreve a seguir. 
Fórmula para definição do recurso de orçamento a ser recebido por uma biblioteca (3)

$$
R_{i}=\frac{k_{i} c_{i} \sum_{j} p_{j i}}{\sum_{i} k_{i} c_{i} \sum_{i} \sum_{j} p_{j i}} A
$$

Onde:

$\sum_{j}$ é o somatório sobre todas as obras de uma biblioteca "i";

$\sum_{i}$ é o somatório sobre todas as bibliotecas;

$R_{i}$ é o recurso a ser recebido pela biblioteca "i";

A é o valor total do orçamento a ser distribuído;

$k_{i}$ é o preço médio dos livros da área da biblioteca “'i”;

$c_{i}$ é a distância percentual do acervo da biblioteca

“i” em relação ao acervo ideal necessário para o atendimento da demanda. Este dado é obtido como segue:

$c_{i}=\frac{\sum_{j=1} p_{j i}}{\sum_{j=1} t_{j i}}$
Na tabela 2 mostramos dados fictícios para exemplificar a aplicação da fórmula 3. O exercício foi proposto tendo em vista a tarefa imaginária de distribuição de cem mil reais $(\mathrm{R} \$ 100.000,00)$ entre as oito bibliotecas mencionadas na tabela.

De acordo com as informações a tabela 2, existem oito (8) bibliotecas de áreas diferentes que se encontram em situações distintas em termos de acervo para o atendimento da demanda potencial por livros didáticos de cursos de graduação. Observa-se, por exemplo, que a Biblioteca de Engenharia possui o maior número de carências absolutas no seu acervo, ao contrário da Agronomia que apresenta a menor quantidade. E ainda, tendo em vista o acervo ideal que as bibliotecas deveriam ter para atender sua comunidade, a Biblioteca de Biociências encontra-se percentualmente mais distante do esperado: precisaria de um incremento de $83 \%$ no número de exemplares para possuir uma coleção plenamente satisfatória.

Além das carências de acervo das bibliotecas, consideradas de forma absoluta e relativa, a fórmula contempla as diferenças existentes no preço médio dos livros das várias áreas. De outro modo, a capacidade de investimento das unidades que trabalham com materiais tradicionalmente mais caros seria prejudicada.

TABELA 2

Parâmetros para distribuição de recursos entre as bibliotecas do SBU

\begin{tabular}{|c|c|c|c|c|c|}
\hline Biblioteca & $\begin{array}{l}\text { N. ideal de } \\
\text { exemplares }\end{array}$ & N. de Carên cias & \% de Carências & Preço médio & $\begin{array}{c}\text { Recurs o a } \\
\text { receber }\end{array}$ \\
\hline $\mathbf{i}$ & $\Sigma \mathbf{t}_{\mathrm{i}}$ & $\Sigma p_{i}$ & c & k & $\mathbf{R}$ \\
\hline Agronomia & 840 & 455 & 0,54 & 144,27 & $2.818,16$ \\
\hline Artes & 1.189 & 649 & 0,55 & 62,80 & $1.763,25$ \\
\hline Biociências & 1.916 & 1.594 & 0,83 & 206,45 & $21.699,14$ \\
\hline Economia & 3.240 & 2.355 & 0,73 & 117,68 & $15.965,63$ \\
\hline Enfermagem & 739 & 538 & 0,73 & 115,55 & $3.587,04$ \\
\hline Engenharia & 3.468 & 2.447 & 0,71 & 245,51 & $33.597,32$ \\
\hline Farmácia & 938 & 564 & 0,60 & 260,45 & $7.000,46$ \\
\hline Fisica & 1.958 & 1.251 & 0,64 & 214,19 & $13.569,00$ \\
\hline Total & 14.288 & $9.853,00$ & 5,32 & $1.366,90$ & $100.000,00$ \\
\hline
\end{tabular}


O método BiblioGrad para avaliação de acervos de livros de graduação: instrumento para gestão de recursos para ...

Um exemplo interessante para ilustrar este problema é dado pela comparação das informações das bibliotecas de Economia e de Engenharia, na tabela 2. Ambas possuem carências absolutas e relativas semelhantes e deveriam receber quantidade de recursos praticamente idêntica, caso o preço médio dos livros não fosse observado. Entretanto, se assim o fosse, seria bastante diversa a capacidade de incremento dos dois acervos com o mesmo recurso, pois os livros de engenharia custam em média aproximadamente o dobro dos de economia. Como a fórmula 3 congrega esses diferentes aspectos, a engenharia em nosso exemplo ganha igualmente aproximadamente o dobro de recursos do que a economia. Todavia, estima-se que ambas conseguirão comprar com valores a elas dedicados praticamente a mesma quantidade de exemplares de livros, 136 aproximadamente.

Para finalizar, a figura 4 ilustra a colocação dos dados da tabela 2 para a realização dos cálculos.

\section{O N S I ERA ÇÕ ES F INA I S E PERSPECTIVAS DE APERFEIÇOAMENTO DO MÉTODO}

A Biblioteca Central já utilizou o BiblioGrad para a distribuição de orçamentos entre as bibliotecas setoriais por quatro ocasiões. Preliminarmente, temos observado que o método parece cumprir com a finalidade para a qual foi idealizado. Todavia, estamos coletando os resultados de todas as avaliações realizadas nos diferentes processos de distribuição de recursos para acompanhar em que medida o potencial de investimento determinado pelo BiblioGrad tem contribuído para que as diferentes bibliotecas tenham sua capacidade de atendimento das necessidades por livros para o ensino de graduação relativamente uniformizada e, a longo prazo, significativamente melhorada.

Pretendemos ainda desenvolver o método no sentido de produzir relatórios mais adaptados

FIGURA 4

Exemplo de aplicação da Fórmula 3 com dados da Biblioteca de Agronomia

\begin{tabular}{|c|c|c|c|c|c|c|}
\hline Biblioteca & $\begin{array}{l}\text { N. ideal de } \\
\text { exemplares }\end{array}$ & $\begin{array}{c}\text { N. de } \\
\text { Carências }\end{array}$ & \% de Carências & $\begin{array}{l}\text { Preço } \\
\text { médio }\end{array}$ & $\begin{array}{c}\text { Fator de } \\
\text { normalizaçao }\end{array}$ & $\begin{array}{l}\text { Recurs o a } \\
\text { receber }\end{array}$ \\
\hline $\mathbf{i}$ & $\mathbf{u}_{i}$ & $\Sigma \mathbf{p}_{i}$ & c & k & & $\bar{R}$ \\
\hline Agronomia & 840 & 455 & 0,54 & 144,27 & 35.556 .54 & $2.818,1$ \\
\hline Artes & 1.189 & 649 & 0,55 & 62,80 & $22.246,78$ & $1.763,25$ \\
\hline Biociências & 1.916 & 1.594 & 0,83 & 206,45 & 273.776 .41 & 21.699 .1 \\
\hline Economia & 3240 & 2.355 & 0,73 & 117,68 & $201.437,11$ & $15.965,6$ \\
\hline Enfermagem & 739 & 538 & 0,73 & 115,55 & 45.257 .45 & 3.587. \\
\hline Engenharia & 3.468 & 2.447 & 0,71 & 245,51 & $423.894,75$ & $33.597,32$ \\
\hline Farmácia & 938 & 564 & 0,60 & 260,45 & $88.324,20$ & $7.000,4$ \\
\hline Fisica & 1.958 & 1.251 & 0,64 & 214,19 & $171.198,96$ & $13.569,00$ \\
\hline Total & 14.288 & $9.853,00$ & 5,32 & $1.366,90$ & $1.261 .692,20$ & $100.000,00$ \\
\hline
\end{tabular}

Recurso(A)

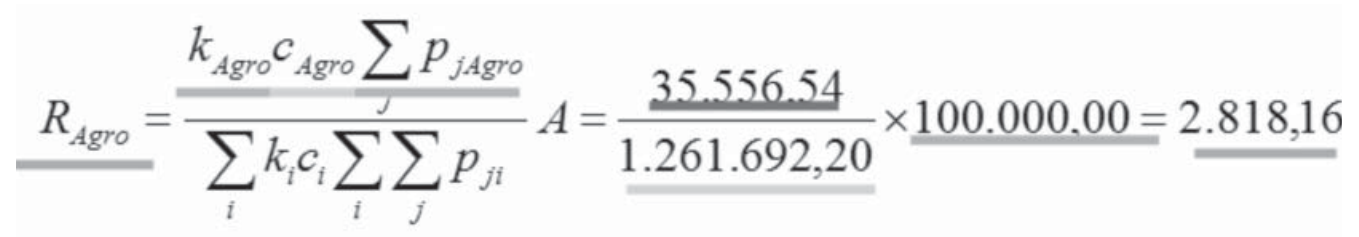


às necessidades das bibliotecas setoriais em sua tarefa de seleção dos títulos a serem adquiridos. Pelo retorno dado pelas bibliotecas, verificamos que o BiblioGrad já tem qualificado a atividade de seleção, mas os dados ainda não são suficientemente específicos para revelar a realidade própria de cada unidade de ensino. O único relatório de que dispomos, descrito na tabela 1 , não distingue as carências por departamento ou curso atendido pelas obras citadas nos planos de ensino, nem ao menos identifica os títulos relacionados com disciplinas obrigatórias ou eletivas. A importância dessas informações sempre foi reconhecida por nós, mas ainda estamos tentando superar algumas limitações técnicas do sistema para aperfeiçoar o BiblioGrad neste setor.

Outro aspecto que também nos parece carecer de aperfeiçoamentos são os parâmetros utilizados para a estimativa da demanda potencial. Para calculá-la, partimos da definição geral dada pelo MEC do número de exemplares por aluno ou disciplina (BRASIL, 2008) e aplicamos essa relação aos dados específicos de matrículas dos alunos e às obras do SBU. Todavia, não sabemos até que ponto todos os acervos podem ser analisados de forma adequada com um parâmetro único, independentemente da área.

A variação no tamanho das bibliografias por si só sugere que há uma diferença na forma de uso dos livros nas disciplinas de cursos de áreas diversas. Isso nos faz pensar que a relação aluno/exemplar de uma obra não deve ser a mesma para todos. Assim, uma hipótese que se cogita é a de que, em ramos do conhecimento que costumam ter disciplinas com bibliografias menores, os alunos fazem uso mais intenso dos poucos livros citados. Para esses casos, supomos que as bibliotecas precisariam dispor de uma quantidade maior de exemplares por aluno.

Ao contrário, em disciplinas de áreas com bibliografias extensas, os alunos dispersam sua atenção entre o estudo de várias obras no decorrer do semestre. Para bem atender os usuários nessas circunstâncias, imaginamos que as bibliotecas deveriam ter uma variedade maior de títulos, com uma quantidade relativa menor de duplicações. Entretanto, essas suposições não encontram respaldo nos parâmetros de avaliação de acervos do MEC, mas, pela inexistência de estudos específicos, também não podem ser descartadas.

Neste sentido, estamos com um projeto em andamento na Biblioteca Central da UFRGS para melhor identificar os padrões de uso dos livros de graduação nas diversas áreas e, assim, buscar a consolidação de um conhecimento a respeito dos critérios que possam revelar a estimativa da demanda potencial deste tipo de material.

Por fim, queremos destacar a importância de empreendermos iniciativas no sentido de compilar as estatísticas que revelem os padrões de oferecimento de produtos e serviços nas bibliotecas universitárias brasileiras. Com dados mais específicos sobre a realidade das diversas bibliotecas, vislumbramos a possibilidade de criação de métodos mais específicos, que congreguem um número mais significativo de variáveis capazes de captar as diferenças de necessidades e usos da informação nas várias áreas do conhecimento a serem atendidas.

Artigo submetido em 05/09/2009 e aceito em 06/05/2011.

\section{REFERÊNCIAS}

BRASIL. Ministério da Educação. Instrumento de avaliação dos cursos de graduação: 2008. Disponível em: < http://www.inep.gov. br/download/condicoes_ensino/2008/Instrumento_Avaliacao_ Cursos_v_final_9setembro.pdf >. Acesso em: 17/11/2008.

BUDD, J. M. Allocation formulas in the literature: a review. Library Acquisition: practice and theory, v.15, n.1, p.95-107, 1991.

CANEPI, K. Fund allocation formula analysis: Determining elements for best practices in libraries. Library Collections Acquisitions \& Technical Services, v.31, n.1, p.12-24, 2007.

DIAS, Maria Matilde Kronka; PIRES, Daniela. Formação e desenvolvimento de coleções de serviços de informação. São Carlos: EdUFSCar, 2003. (Serie apontamentos).

FIGUEIREDO, Nice Menezes de. Desenvolvimento \& avaliação de coleções. 2.ed. rev. e atual. Rio de Janeiro: Thesaurus, 1998.

GONZAGA FILHA, Zely Siqueira et al. Compra automática de bibliografias de cursos, adequadas às exigências do MEC.

Ci. Inf., Brasília, DF, v. 39 n. 3, p.105-115, set./dez., 2010 
O método BiblioGrad para avaliação de acervos de livros de graduação: instrumento para gestão de recursos para ...

IN: SEMINÁRIO NACIONAL DE BIBLIOTECAS UNIVERSITÁRIAS,14., 2006, Salvador. Anais. 2006.

LANCASTER, F. Wilfrid. Avaliação de serviços de bibliotecas. Brasília: Briquet de Lemos/Livros, 1996.

LUBISCO, N. A. A biblioteca universitária brasileira: uma proposta para avaliar seu desempenho. Ponto de Acesso, v. 2, n. 1, p. 153-199, 2008.

SPILLER, David. Book selection : principles and practice. 5.ed. London: Library Association, 1991.

STREHL, Leticia; CASTANHO, Viviane C. BiblioGrad: metodologia para avaliação do acervo de graduação. Disponível em: <http://www.biblioteca.ufrgs.br/modulo8-3.ppt>. Acesso em: 07/04/2011.
UNIVERSIDADE DE SÃO PAULO. Sistema Integrado de Bibliotecas. Subsídios para o estabelecimento de política de desenvolvimento de acervos para as bibliotecas do SIBi/USP. São Paulo: Sibi/USP, 1998. (Cadernos de estudos; 7).

VERGUEIRO, Waldomiro. Desenvolvimento de coleções. São Paulo: Polis, 1989. (Palavra-chave).

VICENTINI, Luiz A. et al. Aquisição de livros de graduação na UNICAMP : política de alocação e divisão de recursos. IN: SEMINÁRIO NACIONAL DE BIBLIOTECAS UNIVERSITÁRIAS,14., 2006, Salvador. Anais. 2006.

WALTERS, W. H. A regression-based approach to library fund allocation. Library Resources \& Technical Services, v.51, p.263-278, 2007. 